\title{
ANÁLISE ACÚSTICA E PERCEPTIVO-AUDITIVO DA VOZ DE MULHERES ADULTAS DURANTE O CICLO MENSTRUAL
}

\author{
Debora Godoy Galdino, Maria Edilaine Alves Roncolato, Patrícia Arruda de Souza Alcarás \\ Universidade do Oeste Paulista - UNOESTE, Curso de Fonoaudiologia, Presidente Prudente, SP. E-mail: \\ deboragodoy@unoeste.br
}

\section{RESUMO}

O objetivo do presente estudo foi analisar os efeitos das alterações hormonais na voz em mulheres durante o ciclo menstrual. Participaram do estudo 12 mulheres, com idade média de 22 anos. Para a avaliação perceptivo-auditiva da voz foi utilizado o protocolo CAPE-V. Para analise acústica foi utilizado o programa VOX METRIA. Os resultados da comparação da análise acústica não houve diferença estatisticamente significante. Porém, foi possível observar variação nos valores da frequência fundamental (F0). Quanto à Jitter e shimer, tiveram seus valores diminuídos no início da menstruação e ao final ocorreu um aumento significativo. Na análise perceptivo-auditiva, observou-se um aumento estatisticamente significante nos valores das variáveis, grau geral e tensão. Concluiu-se, que no período menstrual há mudanças na qualidade vocal, sendo estas, redução da frequência fundamental, shimmer e jitter no primeiro dia da menstruação se comparado aos demais, bem como, aumento estatisticamente significante nos valores de grau geral e tensão.

Palavras-chave: voz, qualidade vocal, menstruação, acústica da fala, distúrbios da voz.

\section{ACOUSTIC AND PERCEPTUAL AUDITORY VOICE ANALYSIS OF ADULT WOMEN DURING THE MENSTRUAL CYCLE}

\begin{abstract}
The aim of this study was to analyze the effects of hormonal changes in the voice in women during the menstrual cycle. The study included 12 women with a mean age of 22 years. For the perceptual evaluation of voice was used CAPE-V protocol. For acoustic analysis was used VOX Metria program. The results comparing the acoustic analysis revealed no statistically significant difference. However, it observed variation in the values of the fundamental frequency (F0). As for Jitter and shimer, they had their values diminished early in the period and at the end there was a significant increase. The perceptual analysis, there was a statistically significant increase in the values of the variables, general degree and tension. It was concluded that the menstrual period there are changes in vocal quality, being these, reducing the fundamental frequency, jitter and shimmer on the first day of menstruation compared to others, as well as a statistically significant increase in overall grade and voltage values.
\end{abstract}

Keywords: Keywords: voice, vocal quality, menstruation, voice quality, voice disorders

\section{INTRODUÇÃO}

A menstruação é um fenômeno biológico que ocorre na puberdade por volta de 12 a 13 anos de idade, ocorrendo uma série de mudanças físicas e mentais na vida das mulheres. É definida por períodos irregulares, com a quantidade e duração do fluxo sanguíneo podendo variar de ciclo em ciclo. Nos primeiros dias da menstruação é comum a presença de sinais como dores de cabeça, cólicas abdominais e dores nas costas.
Os principais hormônios sexuais femininos, o estrógeno e progesterona, o estrógeno é produzido pelo hormônio folículoestimulante (FSH) sendo responsável por estimular as células da granulosa do folículo ovariano, e a progesterona é produzida pela transformação do folículo ovariano em corpo lúteo.

Durante o ciclo menstrual, com duração média de 28 dias, os níveis de hormônios 
estrógeno e progesterona se variam, resultando evidentemente em fases ${ }^{1}$. A fase menstrual é definida como o primeiro dia do sangramento, a fase proliferativa é responsável pelo crescimento dos folículos ovarianos, e controlada pelo estrogênio que estes folículos secretam, a fase lútea é responsável pela formação, crescimento e funcionamento do corpo lúteo'. Os hormônios causam alterações na qualidade vocal, pois durante o ciclo menstrual as mulheres sofrem vários desconfortos, apresentando sintomas como irritabilidade, estresse, ansiedade, insônia dentre outros fatores, podendo ocorrer modificações na qualidade vocal, pois a voz sofre muita influência de hormônios e de emoções ${ }^{2,3}$.

A voz é um elemento de comunicação primária e de fundamental importância, o elemento qual o ser humano se dispõe para interagir com o próximo. Por meio dela observam-se todas as emoções que o indivíduo transmite, podendo interferir de modo decisivo na eficácia da comunicação social ou profissional.

Uma voz considerada de qualidade equilibrada é aquela que se apresenta de forma agradável, sem esforço durante a emissão que é flexível e não causa nenhum desconforto para o ouvinte. E qualquer alteração na qualidade vocal pode prejudicar a comunicação e a produção natural da voz, sendo essencial se ter uma boa qualidade vocal para assim se ter uma vida mais saudável e para isso é necessário conhecer tantos os fatores que colaboram para uma boa qualidade vocal como para aqueles fatores que prejudicam a qualidade da voz, para prevenção e permanência de uma saúde vocal de qualidade ${ }^{4}$.

Muito se tem estudado sobre os profissionais que utilizam a voz como mecanismo de trabalho, pois é necessária a boa qualidade vocal para que a mensagem que está sendo transmitida seja compreendida pelo interlocutor sem causar algum desconforto. Várias são as exigências feitas para o profissional qualificado se enquadrar no competitivo mercado de trabalho, podendo considerar que a voz faz parte dessas exigências, pois a qualidade vocal é um dos principais fatores que ocasionam sucesso na vida pessoal e profissional. São necessários cuidados especiais com a voz para, desta maneira, evitar hábitos inadequados para a qualidade vocal.

Ressalta-se a importância de se pesquisar sobre a voz das mulheres durante o ciclo menstrual e as mudanças que ocorrem na mesma e ressaltar os cuidados com a voz por meio da higiene vocal e a orientação quanto aos fatores que afetam a qualidade vocal.

O objetivo deste estudo foi observar as possíveis alterações vocais durante o ciclo menstrual, identificar quais as modificações que ocorrem na voz, mediante a influência de hormônios, quando esta diferença está presente e se é observada pelas mulheres, com o intuito de orienta-las a prevenir hábitos inadequados que prejudicam a voz.

\section{METODOLOGIA}

Esta pesquisa foi de caráter, exploratório e quali-quantitativo. Foi submetido ao Comitê de Ética em Pesquisa (CEP), sob o número do CAAE 33843014.8.0000.5515 e Comitê Assessor de Pesquisa Institucional (CAPI), sob o número 2192 da Universidade do Oeste Paulista de Presidente Prudente - SP, tendo sido realizado somente após sua aprovação.

O desenvolvimento deste trabalho, contou com a participação de mulheres adultas que foram esclarecidas e informadas sobre todos os procedimentos a que foram realizados assim como os objetivos do trabalho. Foi solicitada a assinatura do Termo de Consentimento Livre e Esclarecido - TCLE. Todos os princípios éticos em pesquisa com seres humanos contidos no Art. 290 do código de ética do Fonoaudiólogo e resoluções CNS 466/12 foram respeitados no decorrer da pesquisa.

Participaram deste estudo 12 mulheres voluntárias, com idades entre 20 a 27 anos. 0 critério para exclusão das participantes foi apresentar queixas vocais, ser fumante e fazer uso de contraceptivos orais.

As participantes recrutadas para a pesquisa não possuiam vínculo com qualquer instituição. Estas mulheres foram convidadas pela própria pesquisadora e só participaram da pesquisa as que quiseram colaborar voluntariamente, podendo desistir da mesma a qualquer momento.

As participantes tiveram sua voz avaliada e, para aquelas com alguma alteração, houve orientação quanto ao diagnóstico e tratamento. As mulheres participantes foram beneficiadas com o estudo, pois tiveram sua voz avaliada por especialista. Foi realizada anamnese vocal para descrever melhor o perfil vocal da participante e detectar se a mesma apresentava alterações na voz e no ciclo menstrual. A voz de cada participante foi gravada no primeiro dia da menstruação, no final da menstruação e por volta 
do décimo quarto dia após o início da menstruação. As vozes foram analisadas por meio da análise perceptivo-auditiva e acústica da voz.

As gravações das vozes das participantes foram realizadas numa clínica escola de Fonoaudiologia de uma instituição de ensino superior no interior do Estado de São Paulo. A gravação foi realizada com a participante sentada com tronco reto, com as costas apoiadas no encosto da cadeira, braços e mãos relaxados sobre as pernas. Para a gravação dos sinais de voz, foram solicitadas ao indivíduo as seguintes emissões: vogais /a/ e /é/ sustentadas em frequência e intensidade confortável, leitura das frases contidas no protocolo CAPE- $V$ e fala espontânea.

A avaliação perceptivo-auditiva foi realizada por fonoaudiólogo treinado e foi utilizado o protocolo CAPE-V, validado pela literatura, que teve como objetivo avaliar a qualidade vocal por meio de uma marcação em uma escala visual-analógica de $100 \mathrm{~mm}$, cuja interpretação constou da análise do canto esquerdo da régua (0), que corresponde a ausência de alteração e o extremo direito (100) que se refere às alterações severas de cada parâmetro analisado. Esta escala foi usada para avaliar a qualidade da voz considerando os seguintes aspectos: grau geral, rugosidade, soprosidade, tensão, pitch e loudness, assim como comentários sobre a ressonância.

Para a análise acústica tradicional as medidas utilizadas foram: frequência fundamental (FO), expressa em $\mathrm{Hz}$, jitter e shimmer, expressos em porcentagem, irregularidade e ruído. Para a mensuração dos parâmetros acústicos foi utilizado o programa VOX METRIA, da CTS informática. Para esta análise foi selecionado o trecho de maior estabilidade do sinal de voz, sendo eliminado o início e o final da emissão da vogal sustentada /é/.

Os resultados obtidos neste estudo foram apresentados em tabelas e gráficos para facilitar a análise e a apresentação. Foi utilizada estatística descritiva para os cálculos da média e desvio padrão, medidas máxima e mínima e porcentagens, quando apropriadas.
$\mathrm{Na}$ comparação estatística entre a avaliação perceptivo-auditiva e acústica dos momentos do ciclo menstrual (durante e após) foi utilizado o teste de Friedman, com nível de significância de $p<0,05$. As análises foram realizadas no programa BioEstat 5.3.

O Coeficiente de Correlação Interclasse (CCI) foi utilizado para a análise estatística da confiabilidade das avaliações perceptivo-auditiva, para tanto foram repetidas as avaliações de $20 \%$ das vozes.

Os resultados do Coeficiente de Correlação Intraclasse foram interpretados a partir dos estudos a partir dos estudos publicados em literatura específica ${ }^{6}$, sendo:

a. Valores de menores que 0,40 , concordância pobre;

b. Valores entre 0,40 e 0,75, concordância satisfatória

c. Acima de 0,75, concordância excelente.

\section{RESULTADOS}

Este estudo analisou a voz de 12 mulheres durante o ciclo menstrual, com idades compreendidas entre 20 a 27 anos de idade, sem queixas vocais, não fumantes e sem uso de contraceptivo oral.

Os resultados da análise acústica durante, no final da menstruação e após a menstruação, nas medidas da frequência fundamental (FO) pela média, moda e desvio padrão expressos em Hertz, jitter e shimmer expressos em porcentagem, irregularidade e ruído das mulheres participantes deste estudo estão apresentadas na Tabela 1.

Os resultados da análise perceptivoauditivo pelo protocolo CAPE- $V$ das vozes das mulheres participantes deste estudo durante, no final e após a menstruação, nos parâmetros grau geral (GG), rugosidade (RU), soprosidade (SO), tensão (TE) e instabilidade (INST) na avaliação da vogal sustentada (VO), fala espontânea (E) e leitura das frases (F) contidas no protocolo CAPE$\checkmark$ estão descritas na Tabela 2. Ressalta-se que o parâmetro instabilidade só pôde ser observado na vogal sustentada /a/. 
Tabela 1. Variáveis da análise acústica em mulheres durante, no final da menstruação e 14 dias após a menstruação, assim como valores de $p$ (Teste $t$ ). Média \pm desvio padrão.

\begin{tabular}{ccccc}
\hline \multirow{2}{*}{ Variáveis } & \multicolumn{3}{c}{ Menstruação } & \multirow{2}{*}{$\mathbf{p}$} \\
\cline { 2 - 4 } & Durante & Final & Após & \\
\hline Média F0 & $217,01 \pm 21,59$ & $219,46 \pm 21,29$ & $223,14 \pm 20,36$ & 0,3385 \\
Moda F0 & $217,65 \pm 21,67$ & $220,11 \pm 21,12$ & $223,72 \pm 20,38$ & 0,1738 \\
Desvio F0 & $2,49 \pm 0,64$ & $2,17 \pm 0,87$ & $2,91 \pm 1,13$ & 0,5580 \\
Jitter & $0,87 \pm 0,83$ & $2,80 \pm 7,53$ & $1,07 \pm 0,81$ & 0,7165 \\
Shimmer & $5,18 \pm 3,42$ & $9,15 \pm 16,74$ & $7,65 \pm 4,95$ & 0,0970 \\
Irregularidade & $3,92 \pm 0,82$ & $4,25 \pm 1,80$ & $4,36 \pm 0,73$ & 0,2636 \\
Ruído & $1,10 \pm 0,59$ & $1,03 \pm 0,53$ & $1,29 \pm 0,65$ & 0,1738 \\
\hline
\end{tabular}

Tabela 2. Variáveis da análise perceptivo auditivo em mulheres no primeiro dia do ciclo menstrual, no final da menstruação, e 14 dias após a menstruação, assim como valores de $p$ (Teste $t$ ). Média \pm desvio padrão.

\begin{tabular}{l|c|c|c|c}
\hline \multicolumn{1}{c|}{ Variáveis } & \multicolumn{3}{|c|}{ Menstruação } & p \\
\cline { 2 - 4 } & Durante & Final & Após & \\
\hline Grau geral vogal & $12,75 \pm 6,28^{\mathrm{b}}$ & $18,08 \pm 6,46^{\mathrm{a}}$ & $15,00 \pm 6,81^{\mathrm{ab}}$ & 0,0380 \\
Grau geral espontânea & $10,00 \pm 5,36^{\mathrm{ab}}$ & $14,42 \pm 6,04^{\mathrm{a}}$ & $10,25 \pm 7,39^{\mathrm{b}}$ & 0,0458 \\
Grau geral frases & $10,92 \pm 5,32$ & $14,17 \pm 4,90$ & $11,83 \pm 7,00$ & 0,1738 \\
Rugosidade vogal & $3,50 \pm 3,37$ & $5,58 \pm 4,56$ & $5,42 \pm 3,55$ & 0,5580 \\
Rugosidade espontânea & $3,75 \pm 4,45$ & $6,58 \pm 4,94$ & $3,58 \pm 4,44$ & 0,1032 \\
Rugosidade frases & $3,25 \pm 4,20$ & $6,00 \pm 4,16$ & $4,08 \pm 4,83$ & 0,2476 \\
Soprosidade vogal & $5,58 \pm 4,30$ & $9,08 \pm 2,47$ & $5,83 \pm 4,51$ & 0,1032 \\
Soprosidade espontânea & $2,42 \pm 3,45$ & $6,50 \pm 3,78$ & $3,67 \pm 3,42$ & 0,0681 \\
Soprosidade frases & $3,67 \pm 3,85$ & $6,08 \pm 2,02$ & $3,33 \pm 2,23$ & 0,1441 \\
Tensão vogal & $7,00 \pm 4,18^{\mathrm{b}}$ & $11,83 \pm 6,35^{\mathrm{a}}$ & $10,08 \pm 6,91^{\mathrm{ab}}$ & 0,0195 \\
Tensão espontânea & $4,67 \pm 4,21$ & $9,58 \pm 7,48$ & $6,58 \pm 6,02$ & 0,1502 \\
Tensão frases & $5,58 \pm 4,87$ & $10,08 \pm 6,99$ & $8,42 \pm 6,17$ & 0,0568 \\
Instabilidade vogal & $4,25 \pm 4,11$ & $3,08 \pm 4,21$ & $2,50 \pm 3,40$ & 0,4626 \\
\hline \multicolumn{1}{c}{ Nas vala }
\end{tabular}

Nas variáveis que houve diferenças, letras diferentes indicam diferenças entre os períodos.

O Coeficiente de Correlação Intra-classe (CCI) foi utilizado para a análise estatística da confiabilidade das avaliações perceptivo-auditiva, para tanto foram repetidas as avaliações de $20 \%$ das vozes. Os resultados do Coeficiente de Correlação Intraclasse foram interpretados a partir dos estudos publicados na literatura ${ }^{6}$ sendo: valores de menores que 0,40, corcondância pobre; Valores entre 0,40 e 0,75 , concordância satisfatória e acima de 0,75, concordância excelente. Os valores da Correlação intraclasse, assim como a interpretação estão apresentados na Tabela 3.
A distribuição dos valores da análise acústica pela média da Frequência fundamental F0, Jitter, Shimmer, Irregularidade e Ruído por sujeitos participantes da pesquisa, na primeira gravação (primeiro dia do ciclo menstrual), segunda gravação (durante a menstruação) e terceira gravação (quatorze dias após a menstruação) estão apresentados nas Figuras 1, 2, 3, 4 e 5 . 
Tabela 3. Resultados da avaliação Perceptivo-Auditiva, da correlação Intraclasse (CCl) da análise de confiabilidade e interpretação.

\begin{tabular}{l|c|c}
\hline & Valor do CCl & Interpretação \\
\hline Grau geral vogal & 0,0631 & Ruim \\
Grau geral espontânea & 0,1689 & Ruim \\
Grau geral frases & 0,4452 & Média boa \\
Rugosidade vogal & 0,2572 & Ruim \\
Rugosidade espontânea & 0 & Ruim \\
Rugosidade frases & 0 & Ruim \\
Soprosidade vogal & 0 & Ruim \\
Soprosidade espontânea & 0,3085 & Ruim \\
Soprosidade frases & 0,3021 & Ruim \\
Tensão vogal & 0,4532 & Média boa \\
Tensão espontânea & 0,3925 & Ruim \\
Tensão frases & 0,3538 & Ruim \\
Instabilidade vogal & 0,3916 & Média boa \\
\hline
\end{tabular}

Média da frequência fundamental - F0 - por sujeitos

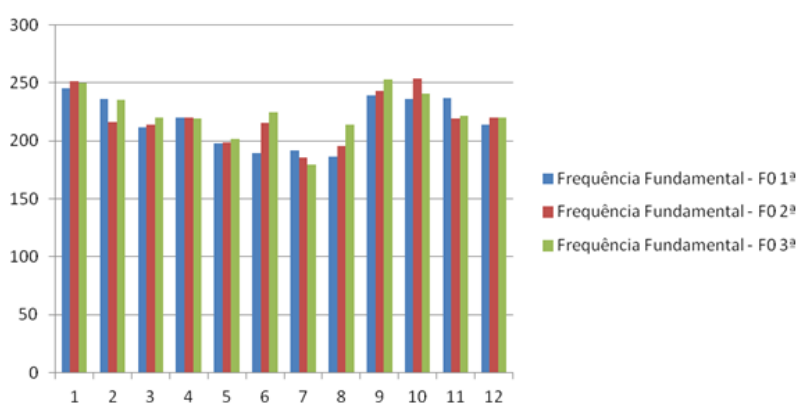

Figura 1. Distribuição da frequência fundamental (F0) das participantes na 1aㅡ. 2a e 3a gravação.

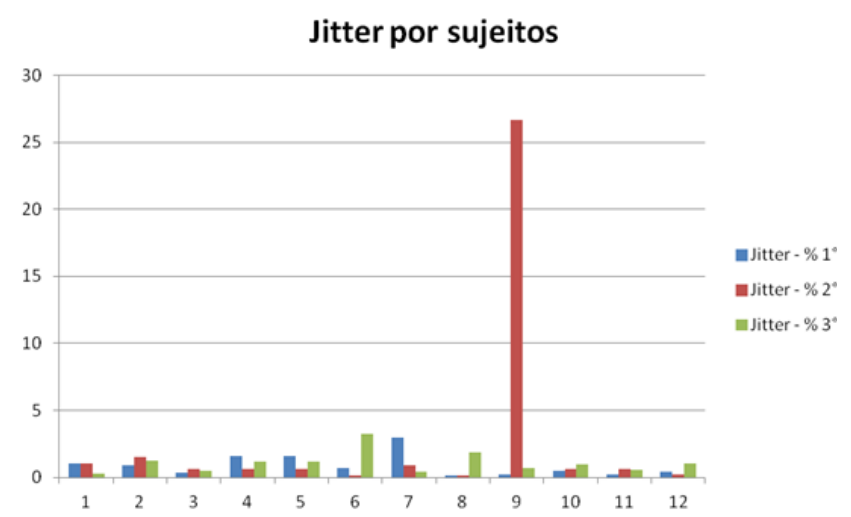

Figura 2. Distribuição do Jitter (\%) das participantes na 1a $2^{2}$ e e 3 a gravação.

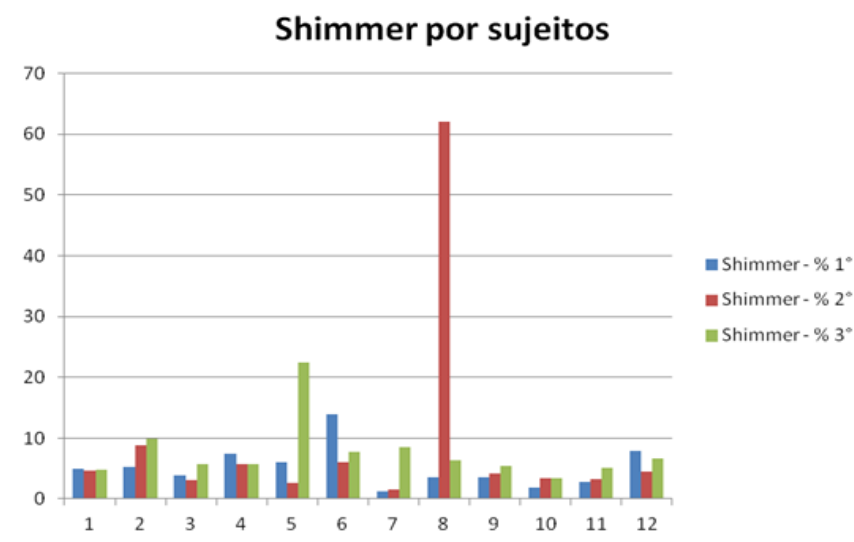

Figura 3. Distribuição do Shimmer (\%) das participantes na 1a , 2a e 3a gravação.

Irregularidade por sujeito

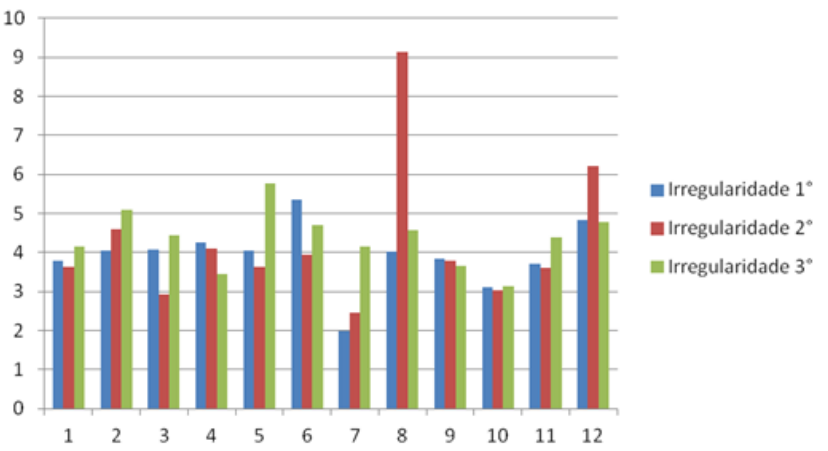

Figura 4. Distribuição da Irregularidade das participantes na 1a , 2a e 3a gravação. 


\section{Ruído por sujeitos}

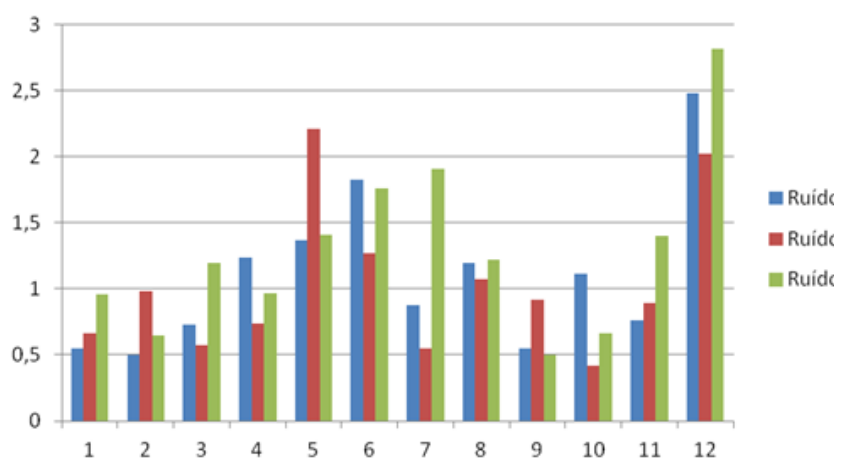

Figura 5. Distribuição do ruído das participantes na 1a 2 2a e 3a gravação.

\section{DISCUSSÃO}

A importância deste estudo centra-se sobre as alterações da voz relacionadas às variações hormonais. A voz é o fator primordial da comunicação e é por intermédio dela que o ser humano se relaciona com o próximo e consegue tocar o outro apenas com a voz, sem necessariamente tocar fisicamente. Sendo uma das extensões mais fortes, que revela aspectos da personalidade, expressa sentimentos e emoções. Por ela ser importantíssima para a comunicação do homem, é indispensável que ela seja saudável. Portanto, é fundamental ter conhecimento dos hábitos inadequados e nocivos à voz. A voz passa por alterações constantemente devido à evolução da idade, saúde física, fatores ambientais, história pessoal de vida e relação de comunicação ${ }^{4}$.

O ciclo menstrual, com período médio de 28 dias, reflete alterações que acontecem no endométrio, sendo os hormônios estrógeno e progesterona, secretados pelos ovários, os causadores. A fase menstrual é caracterizada pelo sangramento que se inicia no primeiro dia e permanece por três a cinco dias, variando de uma mulher para outra ${ }^{1}$.

Neste estudo, analisando os resultados obtidos por meio das análises acústicas da voz, no primeiro dia da menstruação, no final da menstruação por volta do sexto dia e após a menstruação, sendo 14 dias posteriormente ao acontecimento da mesma, foi possível observar que não houve diferença estatisticamente significante nos parâmetros acústicos. Porém, houve variação dos valores da FO nas fases pesquisadas quando comparados nos três períodos. Sendo que, a primeira gravação ocorreu no primeiro dia do acontecimento da menstruação, apresentando o valor da Fo 217,01 $\pm 21,59$, a segunda gravação ocorreu no final da menstruação, sendo em média no sexto dia da menstruação e obteve o valor de Fo 219,46 \pm 21,29 , já a última gravação foi realizada por volta do décimo quarto dia da menstruação após o início da mesma, sendo o valor de Fo 223,14 \pm 20,36 (Tabela 1). Sabendo-se que no período menstrual há presença de edema nas pregas vocais, refletindo nos valores da F0, era previsto a modificação da frequência fundamental nesse período. Bohadana e Pinho ${ }^{7}$ descreveram que a videolaringoestroboscopia mostra pregas vocais com inchaço na terça parte posterior das pregas vocais, fenda posterior, redução do tônus muscular, redução da força de contração do músculo vocal, podendo ser encontrados nódulos vocais bilaterais e assimétricos no terço médio das pregas vocais.

Portanto, o início da menstruação favoreceu a redução da $\mathrm{F} 0$, devido à presença de edema nas pregas vocais, desta forma, as pregas vocais ficam mais pesadas permitindo menos ciclos por segundo, caracterizando assim uma voz mais grave. Entretanto, posteriormente ocorre a drenagem do edema, e isso favorece o aumento da F0, fazendo com que a voz volte a ficar mais aguda, embora essa diferença seja mínima. De acordo com os dados desse estudo, os valores obtidos de FO variaram muito pouco, não indicando assim diferença estatisticamente significante nos parâmetros determinantes da FO nos três períodos estudados. Porém, observou-se que, no início da menstruação a voz se apresenta mais grave e conforme a climatização da menstruação é possível observar uma voz mais aguda. Estas são algumas das alterações vocais, que também foram observadas no estudo ${ }^{2}$.

As medidas analisadas jitter e shimer, comportaram-se de forma similares, com os valores diminuídos no início da menstruação e ao final da menstruação apresentaram-se modificados e com maior valor neste período, quando comparado com os períodos peri e pósmenstrual. Em relação às variáveis ruído e irregularidade, foi observado que seus valores se comportaram simétricos nos três períodos estudados.

É fundamental atentar-se quanto aos distúrbios fonatórios apresentados, pois estes podem ser sintomas secundários de problemas endócrinos. É comum encontrar mulheres com alterações vocais relacionadas aos hormônios 
sexuais. Os principais hormônios são os estrógenos, progesterona e andrógenos ${ }^{7}$.

Quanto à análise perceptivo-auditiva da voz, o estudo avaliou a qualidade vocal por intermédio de uma marcação em uma escala visual-analógica de $100 \mathrm{~mm}$, onde sua interpretação constou da análise do canto esquerdo da régua (0), que corresponde à ausência de alteração e o extremo direito (100) que se refere às alterações severas de cada parâmetro analisado, portanto esta escala avalia diversos aspectos vocais como avalia a qualidade da voz, sendo estes: grau geral, rugosidade, soprosidade, tensão e instabilidade.

Mediante os resultados obtidos na análise perceptivo auditiva por meio do protocolo CAPE-V, que foram representados pela média, desvio padrão, máximo e mínimo, foi possível verificar que os valores obtidos nos períodos estudados (primeiro dia, durante e após a menstruação) tiveram diferentes variações. Em relação às variáveis rugosidade e soprosidade, seus valores foram semelhantes nas três fases analisadas, ocorrendo uma pequena variação. Já quanto os resultados da variável grau geral da voz, observou-se que no final da menstruação houve um aumento estatisticamente significante nos valores da vogal sustentada e fala espontânea. De modo similar, também foi possível verificar um aumento significante na tensão da vogal sustentada no final da menstruação quando comparadas nos três períodos (Tabela 2).

Para confiabilidade estatística das avaliações do CAPE-V, foram repetidas $20 \%$ da amostra dos sinais de voz, que foi avaliada por um fonoaudiólogo da área de voz devidamente treinado, utilizando-se do Coeficiente de Correlação Intra-Classe $(\mathrm{CCl})$, no qual valores menores que 0,40 tiveram concordância pobre, valores entre 0,40 e 0,75 concordância satisfatória e valores acima de 0,75 concordância excelente. Quanto as variáveis analisadas, foi possível verificar que o grau geral das frases e tensão da vogal obtiveram média boa com valores maiores que 0,40 , sendo assim, foi possível verificar uma concordância satisfatória. Em sequência, os aspectos rugosidade, soprosidade e ressonância (instabilidade) apresentaram seus valores inferiores a 0,40 , dessa forma, observou-se concordância pobre (Tabela 3).

$\mathrm{Na}$ aplicação do questionário sobre as possíveis alterações vocais durante o período menstrual, $25 \%$ das participantes referiram sentir cansaço vocal ao falar e apresentar rouquidão, $75 \%$ não percebem alterações vocais durante o período menstrual. Em relação aos sintomas associados durante ciclo menstrual, o estudo revelou que dentre os sintomas questionados os resultados obtidos foram a irritabilidade com $75 \%$, nervosismo com $73 \%$, sensibilidade com $73 \%$, dores musculares com $25 \%$ e todas as ocorrências citadas acima com $0,5 \%$.

\section{CONCLUSÃO}

Sobre o modo como as mulheres vivenciam e enfrentam a Síndrome PréMenstrual, os sintomas característicos e as possíveis consequências e causas que podem interferir na vida social, o estudo revelou que 99\% das mulheres têm a percepção desses sintomas característicos e $81,2 \%$ relatam algum tipo de interferência da síndrome no seu cotidiano. Dentre os sintomas questionados os resultados obtidos foram a ansiedade com $72,4 \%$, a facilidade de choro com $61,4 \%$ e a irritabilidade com $54,4 \%$, foram os mais queixados ${ }^{8}$.

\section{DECLARAÇÃO DE CONFLITO DE INTERESSE}

Todos os autores concordam com a publicação do artigo e declaram que não há conflito de interesses de qualquer natureza.

\section{REFERÊNCIAS}

1. Herliy B, Maebius NK. Anatomia e fisiologia do corpo humano saudável e enfermo. Barueri: Manole; 2002. p.464-5.

2. Figueiredo LC, Gonçalves MIR, Pontes A, Pontes P. Estudo do comportamento vocal no ciclo menstrual: avaliação perceptivo-auditiva, acústica auto-perceptiva. Rev Bras Otorrinolaringol. 2003;70(3):331-9. Doi: https://doi.org/10.1590/S0034-

\section{8}

3. Souza LBR, Monteiro DF, Araujo TRS. Análise acústica da voz falada nos períodos pré e pós menstrual: estudo comparativo. Rev Cienc Med Biol. 2008;7(2):115-22.

4. Behlau M. Voz: o livro do especialista. 2.ed. Rio de Janeiro: Revinter; 2005.

5. Consensus auditory-perceptual evaluation of voice (CAPE-V), ASHA 2003. Rev Soc Bras Fonoaudiol. 2004;9:187-9.

6. Fleiss JL. The Design and analysis of clinical experiments. New York: Wiley; 1986.

7. Bohadana SC, Pinho SMR. Efeitos das alterações hormonais na voz. In: Pinho SMR. 
Fundamentos em Laringologia e voz. Rio de Janeiro: Revinter; 2005. p.165-76.

8. Rodrigues IC, Oliveira E. Prevalência e convivência de mulheres com síndrome prémenstrual. Arq Ciênc Saúde. 2006;13(3):61-7.

Recebido para publicação em 18/12/2015

Revisado em 12/10/2016

Aceito em 14/06/2017 